\title{
Nonequilibrium Corrections to the Spectra of Massless Neutrinos in the Early Universe - Addendum.
}

\author{
A.D. Dolgov 由, S.H. Hansen \\ Teoretisk Astrofysik Center \\ Juliane Maries Vej 30, DK-2100, Copenhagen, Denmark \\ D.V. Semikozt \\ Institute of Nuclear Research of the Russian Academy of Sciences \\ 60th October Anniversary Prospect 7a, Moscow 117312, Russia
}

\begin{abstract}
We repeat our previous calculation of the spectrum distortion of massless neutrinos in the early universe with a considerably better accuracy and corrected for a missing numerical factor in one of the two ways of calculations presented in our paper [1]. Now both ways of calculations are in perfect agreement and we essentially reproduce our old results presented in the abstract of the paper and used in the calculations of light element abundances. We disagree with the criticism of our calculations presented in ref. [2].
\end{abstract}

\footnotetext{
${ }^{1}$ Also: ITEP, Bol. Cheremushkinskaya 25, Moscow 113259, Russia.

${ }^{2} \mathrm{e}-\mathrm{mail}$ : dolgov@tac.dk

${ }^{3}$ e-mail: sthansen@tac.dk

${ }^{4}$ e-mail: semikoz@ms2.inr.ac.ru
} 


\section{Introduction}

In ref. [1] we accurately calculated the distortion of the spectrum of primordial massless neutrinos due to the interaction with hotter electrons and positrons in the primeval cosmic plasma. Our results are in a reasonable agreement with the previous approximate or less accurate calculations (the list of references can be found in ref. [四). Recently a considerably different result was presented in ref. [2]. In view of that, we have redone our calculations with a better accuracy and also corrected for a missing numerical factor in one of the two different but equivalent ways of calculations presented in ref. [1] (see below). Due to this error the results obtained by the two different methods were somewhat different. Now both ways of calculations are in perfect agreement and we essentially reproduced our old results with a better precision政.

The increase in the energy density of neutrinos and the distortion of the spectrum of electronic neutrinos have little effect on light element abundance, but it was recently pointed out that the corresponding increased fraction of cosmic relativistic matter has an impact on the CMB anisotropies, which may be detectable by coming satellite experiments [2, 3].

\section{Description of calculation procedure}

\subsection{Two ways of calculation}

In our previous paper on the subject [1] we used two different approaches for calculating the distortion of the neutrino spectra. We numerically solved the system of equations both for the full neutrino distribution functions, $f_{\nu_{j}}$, and for the deviations from equilibrium, $\delta_{j}=\left(f_{\nu_{j}}-f_{\nu}^{e q}\right) / f_{\nu}^{e q}$. In the last case the contributions to the collision integrals for all the processes vanish for vanishing $\delta$, except for the interactions of neutrinos with electrons, where the "driving force" term, proportional to the temperature difference between $\nu$ and

\footnotetext{
${ }^{5}$ The results quoted in the abstract of ref. [1] as well as the results used in the calculation of $\Delta Y_{H e}$ are the correct ones.
} 


\begin{tabular}{|c||c|c|c|c|c|c|c|}
\hline \multirow{2}{*}{ Program } & points & $a T_{\gamma}$ & $\delta\left(a T_{\gamma}^{e q}\right)$ & $\delta \rho_{\nu_{e}} / \rho_{\nu_{e}}$ & $\delta \rho_{\nu_{\mu}} / \rho_{\nu_{\mu}}$ & $N_{\text {eff }}$ & $\delta N_{\text {eff }}$ \\
\hline \hline \multirow{3}{*}{$\delta(x, y)$} & 100 & 1.399130 & 0.000031 & $0.9435 \%$ & $0.3948 \%$ & 3.03392 & -0.0003 \\
& 200 & 1.399135 & 0.000031 & $0.9458 \%$ & $0.3971 \%$ & 3.03395 & -0.0003 \\
& 400 & 1.399135 & 0.000031 & $0.9459 \%$ & $0.3972 \%$ & 3.03396 & -0.0003 \\
\hline \multirow{3}{*}{$f(x, y)$} & 100 & 1.399079 & -0.000024 & $0.9452 \%$ & $0.3978 \%$ & 3.03398 & 0.0003 \\
& 200 & 1.399077 & -0.000023 & $0.9459 \%$ & $0.3986 \%$ & 3.03401 & 0.0003 \\
& 400 & 1.399077 & -0.000023 & $0.9461 \%$ & $0.3990 \%$ & 3.03402 & 0.0003 \\
\hline \hline
\end{tabular}

Table 1: Two ways of calculation.

$e^{ \pm}$, gives a nonzero contribution. Moreover, for small $x<1$ this contribution is also very small because the temperature difference is approximately $T_{\gamma} / T_{\nu} \approx 1+0.005 x^{2.3}$ which is close to 1 for small $\mathrm{x}$. Here we have defined $T_{\nu}=1 / a$.

However, in the case with the full distribution we erroneously omitted the factor 1.22 (from the Planck mass) for some of the reactions, which created somewhat different results for the two different approaches. Now, after we corrected for this factor, the results are in perfect agreement.

In the recent paper [2] the factor 2 in the rate of the reaction $\nu_{a} \nu_{a} \rightarrow \nu_{a} \nu_{a}$ is missing. Fortunately, the difference in neutrino energy density due to this factor is very small, $\sim 10^{-6}$, which is well below the numerical precision.

We can define the effective number of neutrino species at asymptotically large time as:

$$
N_{\mathrm{eff}}=\frac{\rho_{\nu_{e}}+2 \rho_{\nu_{\mu}}}{\rho_{\nu}^{e q}} \frac{\rho_{\gamma}^{e q}}{\rho_{\gamma}}
$$

where the photon energy density is $\rho_{\gamma}=\left(\pi^{2} / 15\right)\left(a T_{\gamma}\right)^{4}$ and the equilibrium quantities are $\rho_{\nu}^{e q}=(7 / 8)\left(\pi^{2} / 15\right) / a^{4}$ and $\rho_{\gamma}^{e q}=\left(\pi^{2} / 15\right)\left(a T_{\gamma}^{e q}\right)^{4}$.

In the last two columns in tables 1 and 2 we present $N_{\text {eff }}$ from eq. (11). The error on $N_{\text {eff }}$, $\delta N_{\text {eff }}$, comes from the numerical error in the definition of $a T_{\gamma}^{e q}=1.40102$ (see section 2.4). We denote this error $\delta\left(a T_{\gamma}^{e q}\right)$ in tables 1 and 2 . 


\subsection{Initial conditions}

Now, let us discuss the choice of the initial time $x_{i n}$. We made the runs for the system of kinetic equations with three different values $x_{i n}=0.1,0.2$ and 0.5 . We found that the results of the runs with $x_{i n}=0.1$ and $x_{i n}=0.2$ are the same with an accuracy of $10^{-5}$. This means that for $x \leq 0.2$ we can neglect the non-equilibrium corrections to the neutrino distribution functions.

Let us note that already at $x_{i n}=0.1$ the dimensionless photon temperature differs from unity, $a T \neq 1$. For our calculations we took two possible sets of initial conditions. The first was used in the paper [1]:

$$
f_{\nu_{e(\tau)}}=f_{e q}=\frac{1}{e^{y}+1} \quad, \quad a T\left(x_{i n}=0.1\right)=1.00006
$$

These conditions correspond to separate energy conservation in the electromagnetic plasma before the time $x_{i n}$. Note, that even though $a T$ is very close to 1 , we need to keep $a T \neq 1$, because our precision for the equilibrium temperature is of the order 0.00003 . The second set of initial conditions is similar to the one we used in the paper [4]:

$$
f_{\nu_{e(\tau)}}=\frac{1}{e^{y / T}+1} \quad, \quad a T\left(x_{i n}=0.1\right)=1.00003
$$

These conditions correspond to the neutrinos being in thermal equilibrium with the electromagnetic plasma before $x_{i n}$. We found that both initial conditions give the same results for the neutrino energy density and other essential quantities for $x_{i n}=0.1$, i.e. the difference in the results is less than our numerical errors. If one instead would choose $x_{i n}=0.2$ then the condition (3) is more precise.

\subsection{Momentum grid}

We took the dimensionless momentum interval $0 \leq y \leq 20$. For equilibrium neutrinos $\rho_{\nu}(y>20) / \rho_{\nu} \approx 3 \times 10^{-6}$. Because the nonequilibrium correction to the neutrino energy 
density is of the order $1 \%$, neglecting $y>20$ does not affect the result for the neutrino energy density even if the calculation is done with $1 \%$ precision of the effect. There is a somewhat bigger correction in the reaction rates due to the preexponential factor, $\sim p^{2}$, but even for that the momentum cut-off at $y=20$ provides a sufficient accuracy.

In order to choose the distribution of the momentum grid properly let us take a look on the differential energy density of the neutrinos $d \rho_{\nu} / d y=\left(1 / \pi^{2}\right) y^{3} f(y) .97 .5 \%$ of the energy density comes from particles with momentum in the interval $1<y<10$. Particles with momentum $0.1<y<1$ give $1.4 \%$ of the total energy density and particles with $10<y<$ 20 give $1.1 \%$ of the total energy density. Also note that non-equilibrium corrections are particularly important for particles with large momenta.

These arguments advocate the use of a linear distribution in the region $0 \leq y \leq 20$ or $\log (\mathrm{y})$ distribution in the region $0.1 \leq y \leq 20$. We found that the difference between these approaches with the same number of points in grid is about $10^{-6}$ for the neutrino energy density.

The authors of the paper [2] chose $\log (\mathrm{y})$ distribution of points in grid $10^{-5.5}<y<10^{1.7}$ with 40 points per decade. With such a choice more than half of the points lie in the region $y<0.1$, which gives only $0.0002 \%$ contribution to the neutrino energy density. In the most important decade $1<y<10$ they have only 40 points.

In order to check the errors connected with a finite number of points in grid we took the 100, 200 and 400 point grids. The results hereof are presented in table 1.

\subsection{Time evolution}

We used three different methods of time evolution.

1) Euler method. We control the errors connected with a finite number of points in time $x$ in the following way. First, we run the program with some fixed number of points in $x$, distributed in the time interval $x_{i n}<x<x_{f}$ in such a way that the distribution functions 
do not change significantly at any momentum point $y$ during one time iteration $d x$. Then we run the program for the entropy conservation law (i.e. with equilibrium neutrinos) with the same values of time $x_{i}$ as in the first run. Finally we compare the asymptotical values of the temperature ratios with the theoretical value which is $T_{\gamma} / T_{\nu}=(11 / 4)^{1 / 3}=1.40102$. In order to have good precision we require that the numerical error in these temperature ratio should not be larger than $\sim 0.00003$ (fourth column in table 1 ).

2) Bulirsch-Stoer method. Instead of the simple time evolution we used the Bulirsch-Stoer method, described in the book [5].

3) Method for stiff equations. In order to compare our results with the results of the paper [2] we made calculations with their method for stiff equations.

We found that the most precise (in calculation of $T_{\gamma}^{e q}$ ) is the Bulirsch-Stoer method, but in the region of small time $x_{i n}<x<1$ it requires 10 times more processor time than the Euler or the stiff method. Moreover, in the case that we take $x_{i n} \ll 0.1$ only the stiff method takes a reasonably small number of time steps.

In the region of large time $x>1$ the situation is the opposite. The Bulirsch-Stoer method requires 10 times fewer time steps than the Euler method. Unfortunately, we cannot control the precision of the stiff equations method in this time region. The problem is that the energy conservation law, which we use for the evaluation of the photon temperature, is not a stiff equation. In our calculations with the stiff method we therefore evolved the photon temperature as $T_{\gamma}=T_{\gamma}^{e q}+\delta T$. The value of the equilibrium photon temperature $T_{\gamma}^{e q}$ is taken from the entropy conservation equation, while for the evolution of the small $\delta T$ we use the same time steps as we have for the kinetic equations in the stiff method.

In the calculations with the corrections, $\delta(x, y)$, to the distribution functions we used the Euler method. In calculations with the total distribution functions, $f(x, y)$, we used the Euler method for $x<1$ and the Bulirsch-Stoer method for $x>1$. In order to compare our results with the paper [2], we also made the calculations using the stiff method in two 


\begin{tabular}{|c||c|c|c|c|c|c|c|}
\hline Program & points & $a T_{\gamma}$ & $\delta\left(a T_{\gamma}^{e q}\right)$ & $\delta \rho_{\nu_{e}} / \rho_{\nu_{e}}$ & $\delta \rho_{\nu_{\mu}} / \rho_{\nu_{\mu}}$ & $N_{\text {eff }}$ & $\delta N_{\text {eff }}$ \\
\hline \hline $\begin{array}{c}\delta(x, y) \\
\text { Euler }\end{array}$ & 100 & 1.399130 & 0.000031 & $0.9435 \%$ & $0.3948 \%$ & 3.03392 & -0.0003 \\
\hline $\begin{array}{c}f(x, y) \\
\text { Euler+BS }\end{array}$ & 100 & 1.399079 & -0.000024 & $0.9452 \%$ & $0.3978 \%$ & 3.03398 & 0.0003 \\
\hline $\begin{array}{c}f(x, y) \\
\text { BS }\end{array}$ & 100 & 1.399100 & $10^{-7}$ & $0.9463 \%$ & $0.3981 \%$ & 3.03401 & $10^{-6}$ \\
\hline $\begin{array}{c}f(x, y) \\
\text { stiff }\end{array}$ & 100 & 1.399060 & & $0.9518 \%$ & $0.3976 \%$ & 3.03440 & \\
\hline $\begin{array}{c}f(x, y) \\
\text { stiff+BS }\end{array}$ & 100 & 1.399085 & & $0.9399 \%$ & $0.3934 \%$ & 3.03401 & \\
\hline \hline
\end{tabular}

Table 2: Different time evolution algorithms.

different ways: with the stiff method evolution for all $x$, and with a combination of the stiff method for $x<1$ and the Bulirsch-Stoer method for $x>1$. In table 2 we compare the results for these ways of calculation with 100 points grid. We found that all ways of calculation give an effective number of neutrino species around $N_{\text {eff }}=3.0340$, except the stiff method which gives a slightly larger value $N_{\text {eff }}=3.0344$.

\section{Conclusion}

We have seen, that the effect of non-equilibrium neutrinos can be calculated with a very good accuracy if one takes a large enough number of momentum points in the important region $1<y<10$ and a precise algorithm for the time evolution. Finally let us present our results for late times. Photon temperature: $\left(a T_{\gamma}\right)_{\text {final }}=1.39910 \pm 0.00003$, correction to the energy density of electron neutrino: $\delta \rho_{\nu_{e}} / \rho_{\nu_{e}}=0.946 \pm 0.001$, correction to the energy density of muon neutrino: $\delta \rho_{\nu_{\mu}} / \rho_{\nu_{\mu}}=0.398 \pm 0.001$, and effective number of neutrino 
species: $N_{\text {eff }}=3.0340 \pm 0.0003$.

Acknowledgment. We thank Sergio Pastor for pointing out the error of 1.22 in the early version of our program.

The work of $\mathrm{AD}$ and $\mathrm{SH}$ was supported in part by the Danish National Science Research Council through grant 11-9640-1 and in part by Danmarks Grundforskningsfond through its support of the Theoretical Astrophysical Center. The work of DS was supported in part by the Russian Foundation for Fundamental Research through grants 97-02-17064A and 98-02-17493A.

\section{References}

[1] A.D. Dolgov, S.H. Hansen and D.V. Semikoz, "Nonequilibrium Corrections to the Spectra of Massless Neutrinos in the Early Universe", TAC-1997-10; Nucl. Phys. B 503 (1997) 426-444; hep-ph/9703315.

[2] N.Y. Gnedin and O.Y.Gnedin, "Cosmological Neutrino Background Revisited", POP740, astro-ph/9712199.

[3] R.E. Lopez, S. Dodelson, A. Heckler and M.S. Turner, "Precision Detection of the Cosmic Neutrino Background", astro-ph/9803095

[4] Dolgov A.D., Hansen S.H. and Semikoz D.V. "Effect of a massive tau-neutrino on primordial nucleosynthesis. Exact calculations", Preprint TAC-1997-035, 29 p; hep$\mathrm{ph} / 9712284$.

[5] W.H.Press et al., "Numerical Recipes in C: the art of scientific computing", Cambridge U. Press, 1992. 